\title{
A View from The Joint Commission Perspective: Updated Compendium Will Continue to Help Reduce Healthcare-Associated Infections
}

\author{
Margaret VanAmringe, MHS $^{1}$
}

There is a vast body of knowledge generated for the purpose of improving patient outcomes, including information on how to prevent healthcare-associated infections (HAIs). However, it is a daunting task to ensure that the most valuable practices contained within the scientific domain become broadly, timely, and effectively used in practice. Much has been written about the unacceptably long lag time between new clinical knowledge development and its widespread use, even when that knowledge is produced using the gold standard of randomized controlled clinical trials. With this issue in mind, "A Compendium of Strategies to Prevent Healthcare-Associated Infections in Acute Care Hospitals" was written to accelerate adoption of important practices known to be effective at reducing infections. Now updated, enhanced, and included within this and recent issues of Infection Control and Hospital Epidemiology, the Compendium is more than the requisite synthesis of the expansive science on infection prevention and a distillation of that evidence for its relevancy to hospitals. What makes the Compendium unique is its commitment to the usability of its content at the ground level. By presenting information in a format that can make operational sense out of complicated knowledge, the Compendium jump-starts the ability of hospital healthcare professionals to translate essential information into practice.

The Compendium addresses a number of the common impediments to putting new knowledge into daily use. Translating new knowledge into practice requires contending with many barriers and aligning the "Five A's": Awareness, Attitude, Acceptance, Assimilation, and Adoption. First, providers must be aware that the information existsa real challenge with today's volume of published literature and the decreasing time that healthcare professionals can allocate to elective reading. A receptive attitude, or openness to acquiring new information, is a given, but the evidence will only be accepted if it is deemed credible and from a trusted source. The degree to which information is assimilated by healthcare providers depends greatly upon how complex, seemingly contradictory, or otherwise hard to understand, evaluate, and apply the information is; in other words, the new information has to make sense and be considered useful to real-world application. Finally, to be adopted and made operational, new information must be presented in a format and manner that makes it readily implementable and that reduces, to the extent possible, ambiguity about how to use strategies effectively.

The Compendium addresses many of these requisites. Certainly, the source of the information is reputable. The Compendium encompasses current, credible science synthesized by expert vetting into a definitive, single resource for healthcare professionals. Moreover, the review process involved professional organizations renowned for their expertise and dedication to infection prevention and control, working in partnership. Furthermore, the significant industry reach of the Compendium partners will help to ensure broad awareness of its content.

When the Society for Healthcare Epidemiology of America (SHEA) and the Infectious Diseases Society of America (IDSA) Practice Guidelines Committee began convening experts for the Compendium project in 2006, The Joint Commission enthusiastically joined the collaborative effort along with the Association for Professionals in Infection Control and Epidemiology (APIC) and the American Hospital Association (AHA). Experts in the prevention and monitoring of HAIs representing these five organizations formed the HAI Allied Task Force. For both the original and the updated Compendium, professionals serving on the task force worked diligently to review previously published infection control guidelines and recommendations and to discuss, develop, and review compendium content. Their goal was not to duplicate or reevaluate current guidelines but to make the knowledge

\footnotetext{
Affiliation: 1. The Joint Commission, Oakbrook Terrace, Illinois.

Received April 14, 2014; accepted April 16, 2014; electronically published July 16, 2014.

Infect Control Hosp Epidemiol 2014;35(8):964-966

(C) 2014 by The Society for Healthcare Epidemiology of America. All rights reserved. 0899-823X/2014/3508-0004\$15.00. DOI: 10.1086/677147
} 
contained in the science more accessible to clinicians and healthcare organizations.

As a member of The Joint Commission team and a current member of the task force, I can affirm that The Joint Commission greatly valued the opportunity it had to contribute to both the original 2008 and the updated 2014 Compendium. The significant reach of The Joint Commission and our partner organizations in this venture has led to broad dissemination and awareness of the Compendium's content.

From The Joint Commission's view, what makes the Compendium a unique asset to hospitals is the conscious effort behind its development to present the information in an "actionable" format. The Compendium attempts to convey the complex research on infection prevention in a manner that facilitates its use. The goal of its drafting, and the reason for The Joint Commission's involvement as a partner from the inception of the Compendium, was to organize the empirical knowledge in a way that it could be easily employed.

The Compendium partners understood that providers cannot drink from a fire hose of study recommendations and guidelines containing hundreds of potential action steps laced with "shoulds, coulds, mights, musts, and mays" that can confound taking appropriate action. In contrast, the Compendium provides clear and concise directions for preventing HAIs guided by published evidence. The Compendium was designed to make the evidence easy to effectuate and is an indispensable aid to the design of infection prevention programs. The Compendium continues to make important HAIprevention information accessible to busy healthcare professionals; it will be an essential resource toward ongoing efforts by healthcare organizations to drive down HAI incidence.

A Centers for Disease Control and Prevention (CDC) study published in the March 27, 2014, issue of the New England Journal of Medicine indicates that hospitals are making progress in reducing the incidence of HAIs, especially deviceand procedure-related HAIs. ${ }^{1}$ Yet, the CDC's estimate of an HAI reduction from roughly $5 \%$ to $4 \%$ of all inpatients clearly demonstrates the ongoing HAI risks among hospitalized patients and the need for surveillance and prevention of these infections that kill approximately 75,000 Americans each year. $^{2}$

Over the past several years, healthcare organizations throughout America have been able to reduce $\mathrm{HAIs}^{3}$ by applying evidence-based strategies at the patient bedside. Prior to this reduction, HAIs were estimated by the CDC to have afflicted about 1.7 million hospitalized patients each year, ${ }^{4}$ at a cost of nearly 100,000 lives $^{4}$ and up to $\$ 45$ billion annually. ${ }^{5}$ The Compendium has contributed to this reduction by distilling decades of research and literally thousands of guidelines and action steps contained in the scientific literature into a practical resource for clinicians.

Because the Compendium must encourage active intervention against infection, the task force created and has retained a very simple and direct organization for each infection prevention topic-purpose, rationale/concern, detection strategies, prevention strategies, recommendations, and performance measures. The updated Compendium includes a new "examples of implementation strategies" section for all topics to further explain how interventions for each of the HAIs included in the Compendium can be implemented. All recommendations start with action verbs and are written in the imperative to further emphasize the need for urgent action. This focus on actionable information is what sets the Compendium apart from a simple synthesis of the literature.

To further emphasize the importance of HAI prevention, The Joint Commission decided to build on the task force's 2008 published work by developing four National Patient Safety Goals directly related to reducing the risk of HAIs; these goals stem directly from our participation on the task force and focus on implementation of evidence-based practices to prevent (1) central line-associated bloodstream infections (CLABSIs), (2) surgical site infections (SSIs), (3) catheter-associated urinary tract infections (CAUTIs), and (4) HAIs due to multidrug-resistant organisms, such as methicillin-resistant Staphylococcus aureus (MRSA), Clostridium difficile (CDI), and vancomycin-resistant Enterococcus (VRE). ${ }^{6}$ Our task force participation enabled us to quickly gain support for these safety goals. A fifth National Patient Safety Goal relating to HAIs-hand hygiene compliance 6 - preceded our task force participation. Meeting these goals is a requirement for the more than 20,000 healthcare organizations accredited by The Joint Commission.

A new article focused on hand hygiene has been added to the 2014 Compendium updates. The single most important intervention against HAIs-hand hygiene compliance-has long been emphasized by The Joint Commission. Our Center for Transforming Healthcare's Hand Hygiene Project ${ }^{7,8}$ (2008-2009) developed a Targeted Solutions Tool (TST) to guide healthcare organizations through a step-by-step process to measure hand hygiene performance, identify contributing causes to poor compliance, and develop solutions customized to address an organization's particular barriers to hand hygiene improvement. We are pleased that a note has been given in the Compendium to this important work.

A scan of the scientific literature related to HAI prevention demonstrates that the Compendium is often cited, attesting to its usefulness and credibility within both the clinical and academic communities. This result is very gratifying, as the primary goal of the Compendium has been to bridge what is known about HAI prevention strategies based on scientific research and approaches for applying these strategies in actual practice. It has also been very satisfying to see the Compendium translated into two other languages (Spanish and Portuguese) and used to create patient education materials and handy print and digital pocket guides. These formats aid in achieving The Joint Commission's goal of making scientific knowledge more readily available to those who need it and enabling clinicians and others involved in providing health- 
care to address infection prevention challenges and reduce the HAI risks.

\section{ACKNOWLEDGMENTS}

Potential conflicts of interest. The author has no conflicts of interest to disclose relevant to this article.

Address correspondence to Margaret VanAmringe, MHS, The Joint Commission, One Renaissance Boulevard, Oakbrook Terrace, IL 60181 (mvanamringe@jointcommission.org).

\section{REFERENCES}

1. Magill SS, Edwards JR, Bamberg W, et al. Multistate point-prevalence survey of health care-associated infections. $N$ Engl J Med 2014;370:1198-1208.

2. Centers for Disease Control and Prevention. Healthcare-associated infections (HAIs). HAI Prevalence Survey. http://www.cdc.gov /HAI/surveillance/index.html. Accessed March 28, 2014.

3. Centers for Disease Control and Prevention. Winnable battles. Healthcare-associated infections. http://www.cdc.gov /winnablebattles/targets/pdf/hai-winnablebattles -progressreport.pdf. Accessed March 13, 2014.

4. Klevens RM, Edwards JR, Richards CL, et al. Estimating health care-associated infections and deaths in US hospitals, 2002. Public Health Rep 2007;122:160-166.

5. Scott RD. The direct medical costs of healthcare-associated infections in US hospitals and the benefits of prevention. Division of Healthcare Quality Promotion; National Center for Preparedness, Detection, and Control of Infectious Diseases; Coordinating Center for Infectious Diseases; Centers for Disease Control and Prevention, March 2009. http://www.cdc.gov/HAI/pdfs/hai/Scott _CostPaper.pdf. Accessed March 13, 2014.

6. The Joint Commission. National patient safety goals. http:// www.jointcommission.org/standards_information/npsgs.aspx. Accessed March 13, 2014.

7. The Joint Commission Center for Transforming Healthcare. Hand hygiene project. http:/www.centerfortransforminghealthcare.org /projects/detail.aspx?Project =3. Accessed August 21, 2013.

8. The Joint Commission. Measuring hand hygiene adherence: overcoming the challenges. The Joint Commission, Oakbrook Terrace, IL, 2009. http://www.jointcommission.org/assets/1/18 /hh_monograph.pdf. 